\title{
Multi-Objective Genetic Programming for RC Beam Modeling
}

\author{
Amirhessam Tahmassebi ${ }^{1}$, Behshad Mohebali ${ }^{1}$, Anke Meyer-Baese ${ }^{1}$, and Amir Gandomi ${ }^{2}$ \\ ${ }^{1}$ Florida State University \\ ${ }^{2}$ University of Technology Sydney
}

May 6, 2020

\begin{abstract}
This paper presents the application of multi-objective genetic programming in engineering issues. An evolutionary symbolic implementation was developed based on a case study on prediction of the shear strength of slender reinforced concrete beams without stirrups including 1942 set of published test results. In the implementation of the MOGP model, the non-dominated sorting genetic algorithm II with adaptive regression by mixing algorithm with considering the optimization of mean-square error as the fitness measure and the subtree complexity was used. The developed MOGP model was compared to previously developed GP models, different building codes, and additional machine learning-based approaches. It is clearly shown that the MOGP model outperformed the other algorithms applied in this database and can be a general solution to any engineering problems with the main advantage of prediction equations without assuming the prior form of the relevance among the input predictor variables.
\end{abstract}

\section{Hosted file}

AI_Letter_Wiley_MOGP_for_beams_V2.pdf available at https://authorea.com/users/318151/ articles/448088-multi-objective-genetic-programming-for-rc-beam-modeling 